\title{
Adaptive Neural Network Sliding Mode Control for Quad Tilt Rotor Aircraft
}

\author{
Yanchao Yin, Hongwei Niu, and Xiaobao Liu \\ Faculty of Mechanical and Electrical Engineering, Kunming University of Science and Technology, Kunming, Yunnan 650500, China \\ Correspondence should be addressed to Yanchao Yin; yinyc@163.com
}

Received 26 June 2017; Accepted 21 August 2017; Published 11 October 2017

Academic Editor: Guang Li

Copyright (C) 2017 Yanchao Yin et al. This is an open access article distributed under the Creative Commons Attribution License, which permits unrestricted use, distribution, and reproduction in any medium, provided the original work is properly cited.

\begin{abstract}
A novel neural network sliding mode control based on multicommunity bidirectional drive collaborative search algorithm (MCBDCS) is proposed to design a flight controller for performing the attitude tracking control of a quad tilt rotors aircraft (QTRA). Firstly, the attitude dynamic model of the QTRA concerning propeller tension, channel arm, and moment of inertia is formulated, and the equivalent sliding mode control law is stated. Secondly, an adaptive control algorithm is presented to eliminate the approximation error, where a radial basis function (RBF) neural network is used to online regulate the equivalent sliding mode control law, and the novel M-CBDCS algorithm is developed to uniformly update the unknown neural network weights and essential model parameters adaptively. The nonlinear approximation error is obtained and serves as a novel leakage term in the adaptations to guarantee the sliding surface convergence and eliminate the chattering phenomenon, which benefit the overall attitude control performance for QTRA. Finally, the appropriate comparisons among the novel adaptive neural network sliding mode control, the classical neural network sliding mode control, and the dynamic inverse PID control are examined, and comparative simulations are included to verify the efficacy of the proposed control method.
\end{abstract}

\section{Introduction}

It is well known that quad tilt rotors aircraft (QTRA) can be taken as a specific robotic system, whose dynamic modeling and control can be further tailored for further application to generic robotics. In particular, QTRA has been receiving an increasing attention among the global researchers, due to its specific capabilities combined with the advantages of both fixed-wing aerial and helicopters, for example, high cruising speed, long flight range, large loading, and ability of vertical take-off and landing (VTOL) in limited space [1]. Hence, they are more suitable for commercial, firefighting, and investigation purposes, such as assembly of large pieces, natural disasters assessment, reconnaissance of forest fires, and traffic surveillance [2]. However, as a specific robotic manipulators, QTRA is an underactuated system with sixfreedom degree and 4 inputs, which rely on wings and rotors for generating lift; that is, it will have to deal with serious aerodynamic interference between rotors and wings due to their positional relationship [3]. Consequently, the mechanical structure of the QTRA and its strong nonlinearities, unknown external disturbances, coupled dynamics, and multivariable properties, increase the complexity of attitude control designs. In fact, the control design for QTRA has been relatively immature, and any inappropriate control strategy could lead to instability issues, such as the 4 major accidents in the tilt rotor V-22 caused by out of attitude control in the VTOL stage.

With respect to this challenging issue, a considerable amount of effort has been invested for the attitude control of QTRA. In particular, the PID and LQR control methods have been well recognized, where nonlinear structure algorithms are used to suppress integral saturation $[4,5]$, and two linear structure algorithms have been more widely adopted to control integral saturation, including conditional integration [6] and feedback inhibition method [7]. In the subsequent studies, several modifications have been made on the integral part to improve the control capability against saturation, for example, integral part prediction PID modification [8], variable structure PID modification [9], and multimode PID 
modification [10], where the value of integral part will eventually be adjusted with the saturation of controller. However, the majority of these available PID control methods all impose an assumption that the disturbances to be considered are none or varying lowly.

In practical flight scenarios, the uncertainties in the system parameters along the attitude varying may be unavoidable due to complex couplings between the attitude and position, the unknown external disturbances, or the modeling uncertainties and attitude constraints that affect the control system dynamics [11, 12]. Consequently, the precise attitude control is of great importance for the control system design of QTRA. To deal with this issue, some efforts have been advanced to exploit the ability of robustness, invariance to uncertainties, and resistance to external disturbance for sliding mode control (SMC) [13] to improve the robustness of flying craft control. In recent years, many researchers have investigated the incorporation of SMC into adaptive control and intelligent control methods, such as combining SMC with neural networks [14] or with fuzzy logic systems [15]. Particularly, in the application of SMC, its combination with neural network is validated to overcome an obvious shortcoming; that is, the chattering problem that impedes the application of SMC.

It is well known that radial basis function (RBF) neural network can locally approximate unknown dynamic functions with arbitrary precision in certain conditions, which has strong self-learning, adaptation, and fault-tolerant capability $[16,17]$. Accordingly, RBF neural network has been adopted for SMC systems to eliminate chattering phenomenon. It is shown in [18] that the sliding mode variable structure controller using a RBF neural network is more robust to disturbances with a state observer. In $[19,20]$, the application of neural network to estimate the changeable control law and combined boundary layer integral to eliminate the chattering are validated to improve the dynamics of system. It is noted that the presented methods depend on the integral boundary expansion or observer design, where the induced expansionboundary or observer errors are used to reduce the switching frequency of control law for improving the robustness of the traditional sliding mode controller. Moreover, the disturbances and the potential approximation errors are not considered in [21]. Following this framework, the authors of [22] proposed a neural network adaptive backstepping high-order terminal sliding mode control scheme, where neural networks are employed to approximate the unknown nonlinear functions, and a high-order sliding mode control law using the nonsingular terminal is designed to eliminate chattering. Although it was proved that the system is robust to both matched and mismatched uncertainties, the control law of lower-order emerges order coupling during deriving the equivalent control law; that is, the controller is difficult to implement. Besides the observer design and control law regulation, fractional-order calculus theory [23] and discrete time terminal method [24] combing RBF neural network were also exploited to eliminate the chattering of SMC.

In particular, a complementary sliding mode controller is designed by adopting the combination of the generalized sliding surface and the complementary sliding surface to improve the system dynamic performance and robustness [25]. In the latest work, a bioinspired algorithm has attracted researchers to apply such intelligent approaches to optimize the network structure parameters, and the sliding mode error is introduced in the adaptive law to improve the performance of the systems by regulating the centers, widths, and weight of RBF network online. Noticeably, for the attitude control of QTRA with unknown external disturbances and modeling errors, the long convergence time and significant chattering are still a problem to be solved in most of the aforementioned results.

According to the sliding mode control principle, if the system converged to the sliding surface, the performance of sliding mode can be greatly improved. Motivated by this fact and our recent work [26, 27], we proposed a novel adaptive neural network sliding mode control for QTRA attitude with external uncertain disturbances and multichannel couplings, where RBF neural network is used to online regulate the equivalent sliding mode control law, and a robust control law is added to eliminate the approximation error. Then an adaptive control algorithm is designed, where the attitude stability requirements concerning the propeller tension, channel arm, and moment of inertia are all studied. Especially, considering the influence of the hidden layer unit number and the network essential structure parameters on the validity of neural network approximation, a novel multicommunity bidirectional drive collaborative search algorithm is developed to uniformly update the unknown neural network weights and essential model parameters adaptively, where the nonlinear approximation error is obtained and serves as a novel leakage term in the adaptations. Consequently, the attitude of the control system is guaranteed to converge to the sliding surface stably, which can effectively improve the convergence rate and eliminate chattering phenomenon of equivalent sliding mode control law and benefit the overall attitude control performance for QTRA. Appropriate comparisons among the novel adaptive neural network sliding mode control, the classical neural network sliding mode control, and the dynamic inverse PID control are examined. Simulation results are provided to validate the efficacy of the proposed control method.

This paper is organized as follows: the problem to be studied and the attitude dynamics model of QTRA is stated in Section 2. Adaptive neural network sliding mode control with a novel multicommunity bidirectional drive collaborative search algorithm is presented in Section 3. Comparison simulation results are given in Section 4 and conclusion is made in Section 5.

\section{Problem Formulation}

In this section, the proposed QTRA in Figure 1 has 8 inputs of the motors, which includes 4 inputs of regular QTRA and the additional 1 input for each of 4 of the tilting motors, and they are not fixed and are able to tilt-roll separately from each other. In particular, the motors could rotate around pitch to compensate for unexpected effects occurred from internal or external environment. Nevertheless, the body fixed coordinate is defined, where the focal point $O^{b}$ of 


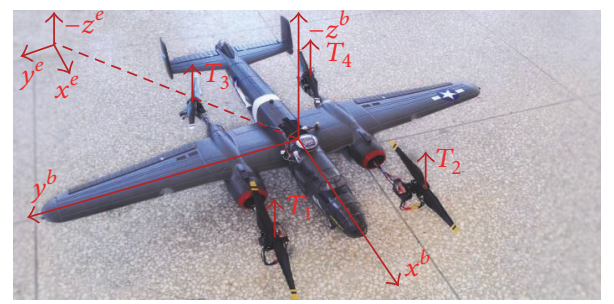

FIGURE 1: Coordinate systems: inertial earth fixed frame and body fixed frame.

gravity for QTRA upwards concurring is chosen as the $Z^{b}$ axis, the perpendicular lever is the $X^{b}$ axis, and the $Y^{b}$ axis is defined by the right hand rule. Then $X^{b}, Y^{b}$, and $Z^{b}$ axes are characterized individually as roll $(\phi)$, pitch $(\theta)$, and yaw $(\varphi)$ angles, respectively. For inertial earth fixed frame, an east-north-up orthogonal coordinate system is created by setting the QTRA flight starting point $O^{e}$ as the origin.
Consequently, a nonlinear attitude control system for QTRA aircraft with attitude angles defined as in Figure 2 is studied, where the QTRA is assumed to be a symmetric rigid body with constant mass, and the roll $(\phi)$ pitch $(\theta)$ and yaw $(\varphi)$ angles have a little variation during the VTOL stage. The transformation matrix $R(\phi, \theta, \varphi)$ from inertial coordinates to body fixed coordinates is given by [23]

$$
R(\phi, \theta, \varphi)=\left[\begin{array}{ccc}
\cos (\varphi) \cos (\theta) & \cos (\varphi) \sin (\theta) \sin (\phi)-\sin (\varphi) \cos (\phi) & \cos (\varphi) \sin (\theta) \cos (\phi)+\sin (\varphi) \sin (\phi) \\
\sin (\varphi) \cos (\theta) & \sin (\varphi) \sin (\theta) \sin (\phi)-\cos (\varphi) \cos (\phi) & \sin (\varphi) \sin (\theta) \sin (\phi)-\sin (\phi) \cos (\varphi) \\
-\sin (\theta) & \cos (\theta) \sin (\phi) & \cos (\theta) \cos (\phi)
\end{array}\right]
$$

If the propeller pull and earth's gravitational force are represented as $T$ and $G$, respectively, in Figure 2 and the resultant force of QTRA body frame is denoted by $F_{i}$, each of the axis thrust vectors $\vec{F}_{i}$ can be given in (2) by using rotation matrix

$$
\begin{aligned}
\vec{F}_{i} & =\left[\begin{array}{c}
F_{i x} \\
F_{i y} \\
F_{i z}
\end{array}\right] \cdot R\left(\phi_{i}, \theta_{i}, \varphi_{i}\right) \\
& =\left[\begin{array}{c}
\left(T_{1}+T_{2}+T_{3}+T_{4}\right) \sin \partial+m g \sin \theta \\
m g \sin \phi \cos \theta \\
\left(T_{1}+T_{2}+T_{3}+T_{4}\right) \cos \partial-m g \cos \phi \cos \theta
\end{array}\right],
\end{aligned}
$$

where $F_{i}, F_{i y}$, and $F_{i z}$ are the thrust force of the components along the axis, respectively, $T_{1}, T_{2}, T_{3}$, and $T_{4}$ are the pull for each of propeller, and $\gamma$ represents the rotor tilting angle.

Suppose $L_{x}, L_{y}$, and $L_{z}$ as the arm length of the rotational torque for $x-, y$-, $z$-axis, respectively; each of the axis torques can be calculated as follows:

$$
\begin{aligned}
& \vec{M}_{i}=\left[\begin{array}{c}
M_{i x} \\
M_{i y} \\
M_{i z}
\end{array}\right] \cdot R\left(\phi_{i}, \theta_{i}, \varphi_{i}\right) \\
& =\left[\begin{array}{c}
\left(T_{1} \cos \gamma+T_{4} \cos \gamma\right) L_{x}-\left(T_{2} \cos \gamma+T_{3} \cos \gamma\right) L_{x} \\
\left(T_{1} \cos \gamma+T_{2} \cos \gamma\right) L_{y}-\left(T_{3} \cos \gamma+T_{4} \cos \gamma\right) L_{y} \\
\left(T_{1} \sin \gamma+T_{4} \sin \gamma\right) L_{z}-\left(T_{2} \sin \gamma+T_{3} \sin \gamma\right) L_{z}
\end{array}\right] .
\end{aligned}
$$

Therefore, the resultant torque of each axis can be calculated, respectively, as follows:

$$
M_{i}=\sqrt{M_{i x}^{2}+M_{i y}^{2}+M_{i z}^{2}} .
$$

According to the law of severity, the torque equation in (4) is produced by the rotation speeds of the propellers, such that (3) becomes

$$
\sum M=\frac{\delta H}{\delta t}+\omega \times H
$$

where $H$ is the moment of momentum of QTRA to the earth frame, and the component of rotation along the angular velocity vector can be given as follows:

$$
\omega=p i+q j+r k .
$$

If the moment of inertia to the body frame is denoted by $I$, the relation between angular momentum and angular velocity can be given as

$$
H=I \omega .
$$

The kinetic equation of centroids for QTRA can be derived in

$$
\begin{aligned}
M_{x}= & I_{x x} \dot{p}-I_{x y} \dot{q}-I_{x z} \dot{r}+\left(I_{z z}-I_{y y}\right) q r \\
& +I_{y z}\left(r^{2}-q^{2}\right)-I_{x z} p q+I_{x y} p r
\end{aligned}
$$



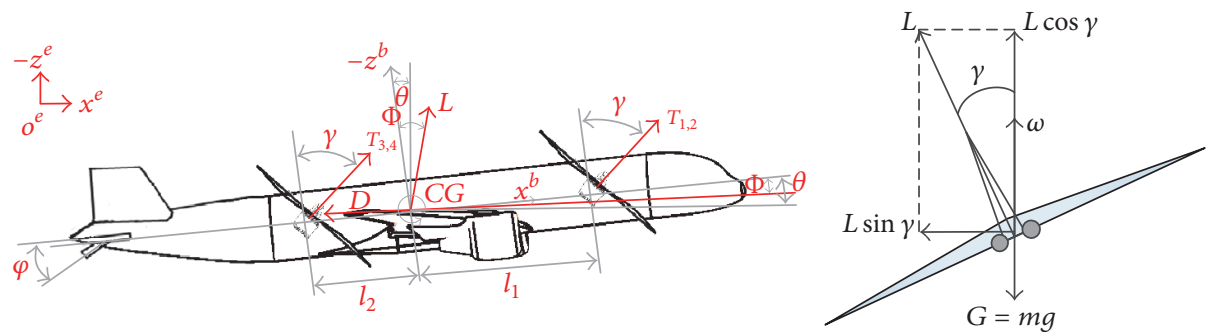

FIGURE 2: Body scheme showing the forces acting on the QTRA.

$$
\begin{aligned}
M_{y}= & -I_{x y} \dot{p}+I_{y y} \dot{q}-I_{y z} \dot{r}+\left(I_{x x}-I_{z z}\right) p r \\
& +I_{x z}\left(p^{2}-r^{2}\right)+I_{z y} p q-I_{x y} q r, \\
M_{y}= & -I_{x z} \dot{p}-I_{z y} \dot{q}+I_{z z} \dot{r}+\left(I_{y y}-I_{x x}\right) p q \\
& +I_{x y}\left(q^{2}-p^{2}\right)+I_{x z} q r-I_{y z} p r,
\end{aligned}
$$

where $I_{x x}, I_{y y}$, and $I_{z z}$ donate the rotary inertia of body frame axis and $I_{x y}, I_{x z}$, and $I_{y z}$ are the inertial products, respectively.

In this study, the roll $(\phi)$ pitch $(\theta)$ and yaw $(\varphi)$ are 1 to 5 degrees, respectively, by the user input during the VTOL stage. In this case we assume that $(\phi, \theta, \varphi)$ equals $(p, q, r)$ and $(\cos \phi, \cos \theta, \cos \varphi)$ equals $(1,1,1)$. Therefore, the attitude dynamics of QTRA can be derived with modeling errors and external disturbances using the formula in (9):

$$
\begin{aligned}
& \ddot{\phi}=\frac{I_{x z}\left(I_{x x}-I_{y y}+I_{z z}\right) \dot{\theta} \dot{\varphi}+\left[I_{z z}\left(I_{y y}-I_{x x}\right)-I_{x z}^{2}\right] \dot{\phi} \varphi+I_{z z} L_{\phi} \partial_{T}\left(\omega_{1}^{2}-\omega_{2}^{2}+\omega_{3}^{2}-\omega_{4}^{2}\right)-I_{x z} L_{\varphi} m g \gamma}{I_{x x} I_{z z}-I_{x z}^{2}}+\Re_{1}, \\
& \ddot{\theta}=\frac{\left(I_{z z}-I_{x x}\right) \dot{\phi} \dot{\varphi}+I_{x z}\left(\dot{\varphi}^{2}-\dot{\phi}^{2}\right)+L_{\theta} \partial_{T}\left(\omega_{1}^{2}+\omega_{2}^{2}-\omega_{3}^{2}-\omega_{4}^{2}\right)}{I_{y y}}+\mathfrak{R}_{2}, \\
& \ddot{\varphi}=\frac{I_{x x}\left(I_{x x}-I_{y y}\right) \dot{\theta} \dot{\varphi}+I_{x z}\left(I_{x x}-I_{y y}+I_{x z}\right) \dot{\phi} \varphi+I_{x z} L_{\phi} \partial_{T}\left(\omega_{1}^{2}-\dot{\omega}_{2}^{2}+\omega_{3}^{2}-\omega_{4}^{2}\right)-I_{x x} L_{\varphi} m g \gamma}{I_{x x} I_{z z}-I_{x z}^{2}}+\mathfrak{R}_{3} .
\end{aligned}
$$

In (11), $\mathfrak{R}_{1}, \mathfrak{R}_{2}$, and $\mathfrak{R}_{3}$ donate composite disturbance for each channel, and in this case we assume that $u_{1}=L_{\phi} \partial_{T}\left(\omega_{1}^{2}-\right.$ $\left.\omega_{2}^{2}+\omega_{3}^{2}-\omega_{4}^{2}\right), u_{2}=L_{\theta} \partial_{T}\left(\omega_{1}^{2}+\omega_{2}^{2}-\omega_{3}^{2}-\omega_{4}^{2}\right)$, and $u_{3}=L_{\varphi} m g \gamma$ stand for control inputs for each channel, respectively, where $\partial_{\mathrm{T}}$ donates tension coefficient of propeller, $\omega_{i}$ is the rotation velocity of propeller, $\mathrm{m}$ is the mass of aircraft, and $L_{\phi}, L_{\theta}$, and $L_{\varphi}$ are the arm of each channel.

Consequently, the nonlinear system of attitude hold control with uncertainties can be written as (12) by defining $x=\left[x_{1}, x_{2}, x_{3}, x_{4}, x_{5}, x_{6}\right]$ as $\hbar=[\phi, \dot{\phi}, \theta, \dot{\theta}, \varphi, \dot{\varphi}]$ using (9)-(11)

$$
\begin{aligned}
& \dot{x}_{(2 k-1)}(t)=x_{(2 k)}(t) \\
& \dot{x}_{(2 k)}(t)=f_{k}(x, t)+\Gamma_{k} u_{k}+\Re_{k} \\
& k=1,2,3,
\end{aligned}
$$

where $u_{k}=\left[u_{1}, u_{2}, u_{3}\right]^{T}, \Re_{k}=\Delta f_{k}(x, t)+\Delta \Gamma_{k} \ell_{k}+\Psi_{k}, \Delta f$ and $\Delta \Gamma_{k}$ represent modeling errors, and $\Psi_{k}$ represents external disturbance,

$$
\Gamma_{k}=\left[\begin{array}{ccc}
\frac{I_{z z}}{I_{x x} I_{z z}-I_{x z}^{2}} & 0 & \frac{I_{x z}}{I_{x x} I_{z z}-I_{x z}^{2}} \\
0 & \frac{1}{I_{y y}} & 0 \\
\frac{I_{x z}}{I_{x x} I_{z z}-I_{x z}^{2}} & 0 & \frac{I_{x x}}{I_{x x} I_{z z}-I_{x z}^{2}}
\end{array}\right] .
$$

\section{Neural Network Sliding Mode Control Optimization Design}

In this section, the design of an adaptive neural network sliding mode controller for QTRA is presented. First, the conventional equivalent sliding mode control for QTRA is designed. Then, the adaptive RBF neural network is proposed based on multicommunity bidirectional drive collaborative search algorithm (M-CBDCS), where the coefficient of switching control is adaptively calculated to overcome the chattering phenomenon of traditional equivalent sliding control. Finally, the stability of the adaptive neural network sliding mode control is proven using Lyapunov theory.

3.1. Equivalent Sliding Mode Control for QTRA. The sliding mode control law based on equivalent control usually consists of an equivalent control law $\mathrm{u}_{\mathrm{eq}_{k}}$ and a switching control law $u_{\mathrm{sw}_{k}}$ which can be obtained:

$$
u_{k}=u_{\mathrm{eq}_{k}}+u_{\mathrm{sw}_{k}}
$$

Conventionally, the equivalent law is deduced from the relationship between the sliding mode $s$ and its differential $\dot{s}$ on the basic of the pertinent mathematic mode of the system, which could ensure the state of system along the sliding surface. The switching control law is applied to compensate 
for uncertainty and external disturbance, which ensures the state of system not leaving the sliding surface.

Considering the nonlinear system about QTRA, the tracking error between the desired reference and the system response is taken as

$$
e_{(2 k-1)}=x_{(2 k-1)}-x_{d(2 k-1)},
$$

where

$$
\dot{e}_{(2 k-1)}=e_{(2 k)} .
$$

According to the theories of sliding mode control, the switching function for the sliding mode can be defined as follows:

$$
s_{k}=c_{k} e_{(2 k-1)}+\dot{e}_{(2 k-1)}, \quad c_{k}>0 .
$$

Combining (12)-(15) and (5), we can get

$$
\begin{aligned}
\dot{s}_{k} & =c_{k} \dot{e}_{(2 k-1)}+\dot{e}_{(2 k)}=c_{k} \dot{e}_{(2 k-1)}+\dot{x}_{(2 k)}-\dot{x}_{d(2 k)} \\
& =c_{k} \dot{e}_{(2 k-1)}-\dot{x}_{d(2 k)}+f_{k}(x, t)+\Gamma_{k} u_{k}+\mathfrak{R}_{k} .
\end{aligned}
$$

According to $\dot{s}_{k}=0$, we can get equivalent control of the sliding mode as follows

$$
\begin{aligned}
& c_{k} \dot{e}_{(2 k-1)}-\dot{x}_{d(2 k)}+f_{k}(x, t)+\Gamma_{k} u_{\mathrm{eq}_{k}}+\mathfrak{R}_{k}=0, \\
& u_{\mathrm{eq}_{k}}=\frac{1}{\Gamma_{k}}\left(-c_{k} \dot{e}_{(2 k-1)}+\dot{x}_{d(2 k)}-f_{k}(x, t)-\mathfrak{R}_{k}\right) .
\end{aligned}
$$

To ensure the sliding mode reaching condition $s(t) \cdot \dot{s}(t) \leq$ $-\eta|s(t)|, \eta>0$, the switching law can be designed as

$$
u_{\mathrm{sw}_{k}}=-\eta \cdot \frac{\operatorname{sgn}\left(s_{k}\right)}{\Gamma_{k}},
$$

where

$$
\operatorname{sgn}\left(s_{k}\right)= \begin{cases}1, & s_{k}>0 \\ -1, & s_{k}<0 .\end{cases}
$$

Remark 1. The switching law shown in (20) is mainly used to compensate for the uncertainties and external disturbances so that system state can retain on the sliding surface and move along it. However, the switching law is actually a nonlinear set of discontinuous functions leading to the chattering problem.

3.2. Adaptive Neural Network Sliding Mode Control. Usually, the equivalent law of sliding mode control depends heavily upon the models and the accuracy of parameters. However, in most cases, these models of complicated system always exist in simplified or linear form by regulating its parameters in different working conditions. Therefore, to compensate for the inaccuracy arising from these uncertainties, the switching law becomes necessary and critical.

In order to overcome the chattering phenomenon of traditional sliding mode control effectively, (20) is replaced by the adaptive switching control law as follows:

$$
u_{\mathrm{sw}_{k}}=-\mu_{N_{k}} \cdot \eta \cdot \frac{\operatorname{sgn}\left(s_{k}\right)}{\Gamma_{k}},
$$

where $\mu_{N_{k}}$ is the adaptive adjusting factor depending on the output of RBF neural network. Thus, this parameter will be optimized using RBF neural network in the following section.

Therefore, we can get the adaptive neural network sliding mode control law as follows:

$$
\begin{aligned}
u_{k} & =u_{\mathrm{eq}_{k}}+u_{\mathrm{sw}_{k}}=\frac{1}{\Gamma_{k}}\left[-c_{k} \dot{e}_{(2 k-1)}+\dot{x}_{d(2 k)}-f_{k}(x, t)\right. \\
& \left.-\Re_{k}\right]-\mu_{N_{k}} \cdot \eta \cdot \frac{\operatorname{sgn}\left(s_{k}\right)}{\Gamma_{k}}=\frac{1}{\Gamma_{k}}\left[-c_{k} \dot{e}_{(2 k-1)}\right. \\
& \left.+\dot{x}_{d(2 k)}-f_{k}(x, t)-\Re_{k}-\mu_{N_{k}} \cdot \eta \cdot \operatorname{sgn}\left(s_{k}\right)\right] .
\end{aligned}
$$

Remark 2. The adaptive neural network sliding mode controller presented in (23) not only inherits the capacity of parameters optimization for M-CBDCS described in Section 3.2.1 but also can identify and estimate the modeling errors $\Delta f, \Delta \Gamma_{k}$ and composite disturbance for each channel $\mathfrak{R}_{k}$.

Proof. In order to demonstrate the stability of the designed controller, consider the following Lyapunov function candidate:

$$
V=\frac{1}{2} s^{2}
$$

Based on (18) and (24), we can obtain the following differential equation:

$$
\begin{aligned}
& \dot{V}=s \dot{s}=s\left[c_{k} \dot{e}_{(2 k-1)}-\dot{x}_{d(2 k)}+f_{k}(x, t)+\Gamma_{k} u_{k}+\Re_{k}\right] \\
& =s\left[c_{k} \dot{e}_{(2 k-1)}-\dot{x}_{d(2 k)}+f_{k}(x, t)\right. \\
& +\Gamma_{k}\left(\frac { 1 } { \Gamma _ { k } } \left[-c_{k} \dot{e}_{(2 k-1)}+\dot{x}_{d(2 k)}-f_{k}(x, t)-\mathfrak{R}_{k}\right.\right. \\
& \left.\left.\left.-\mu_{N_{k}} \cdot \eta \cdot \operatorname{sgn}\left(s_{k}\right)\right]\right)+\mathfrak{R}_{k}\right]=s\left(-\mu_{N_{k}} \cdot \eta\right. \\
& \left.\cdot \operatorname{sgn}\left(s_{k}\right)\right)=-\mu_{N_{k}} \cdot \eta \cdot|s| \text {. } \\
& \dot{V}=s \dot{s} \leq 0 \text {. }
\end{aligned}
$$

Therefore, the stability of adaptive neural network sliding mode controller designed in this paper can be guaranteed when $\mu_{N_{k}}$, the output of RBF neural network, is used to adaptively adjust the switching control law of sliding mode.

3.2.1. Adaptive RBF Neural Network Based on M-CBDCS. For performance and applicability purposes, the M-CBDCS is chosen for optimizing the essential parameters of $\mathrm{RBF}$ network as described in Figure 3. There exist two kinds of communities according to its characteristics in M-CBDCS, where the community searching capacity is evaluated by the cooperation weights and response performance between different communities. Thus, considering the essential parameters optimization problem, the adaptive performance of RBF 


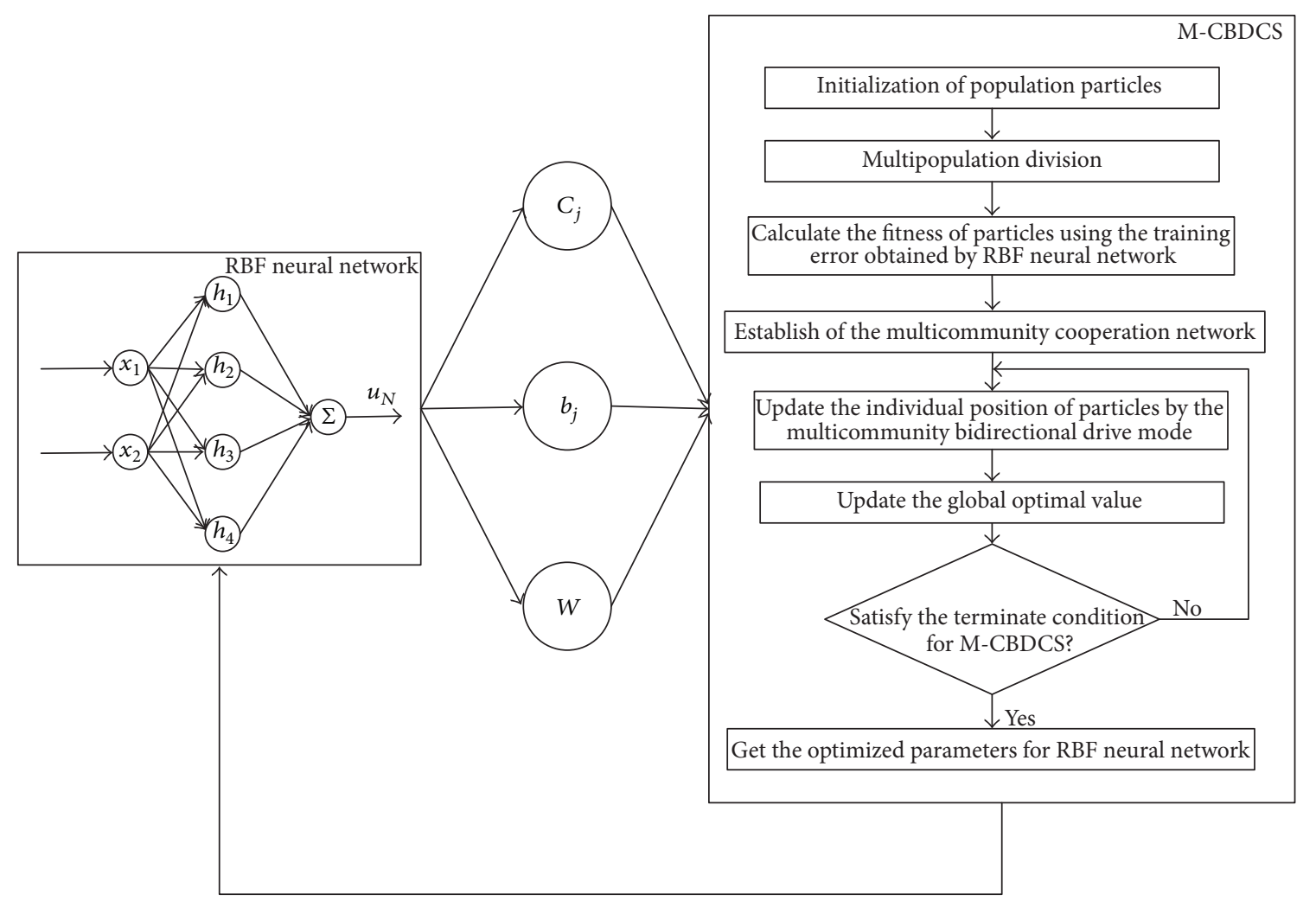

FIgURE 3: The flow diagram about the adaptive RBF neural network with M-CBDCS.

neural network is improved by guiding the evolution of the entire collaboration network from the community with powerful searching ability. Consequently, the center vector $C_{j}$, width vector $b_{j}$, and connection weights $W$ are optimized online adaptively during the RBF neural network training procedure.

Note that RBF neural network has a great advantage of high speed learning and generalization $[28,29]$, which could realize the linearization of nonlinear problem through the transformation of hidden layer. Therefore, the RBF neural network is selected as an approach for switching law adaptive production expressed by (22).

The structure of RBF neural network is shown as follows.

The input layer $X=\left[x_{1}, x_{2}\right]^{T}$ is the vector of two inputs for this network. The input vector is designed to be two elements, which are the sliding mode $s$ and its differential $\dot{s}$.

The hidden layer $H=\left[h_{1}, h_{2}, h_{3}, h_{4}\right]^{T}$ is the vector of activation functions in RBF neural network. The activation function chosen as the Gaussian function is as follows:

$$
h_{j}=\exp \left(\frac{\left\|X-C_{j}\right\|}{2 b_{j}^{2}}\right) \quad j=1,2, \ldots, 4,
$$

where $C_{j}$ is the center vector of this network and $b_{j}$ is the base width vector of this network.

The output layer $\mu_{N}=\sum_{j=1}^{4} \omega_{j} \cdot h_{j}=W^{T} \cdot H$ is used as the adaptive adjusting factor for sliding mode control.
$W=\left[\omega_{1}, \omega_{2}, \omega_{3}, \omega_{4}\right]$ is the vector of weighting factors which connect the hidden layer and the output layer of the network.

Remark 3. For the RBF neural network, the location of hidden unit is determined by the center vector and the size of control area is determined by the width vector. Moreover, the connection weights could directly influence the training time and the output of training as part of the network output. Therefore, the selection of essential parameters including the center vector, width vector, and connection weights is most important to improve the approximation performance of RBF neural network.

In this study a novel M-CBDCS algorithm is presented to uniformly update the essential parameters adaptively for RBF neural network with perfect convergence and high precision. The essential parameters of RBF neural network, including the center vector, width vector, and connection weights, are defined as the particle position vector in particle searching space. Noticeably, the communities are divided into model community (MC) and common community (CC) according to the advantages and disadvantages of community fitness, and the bidirectional interaction mode between communities is constructed as is shown in Figure 4. Under this mode, the member of common community $\mathrm{CC}_{j}$ will be selected into the model community and the worst community in MC will be abandoned according to the bidirectional evolutionary rules. Besides, the learning factor $P_{m}$ is introduced 


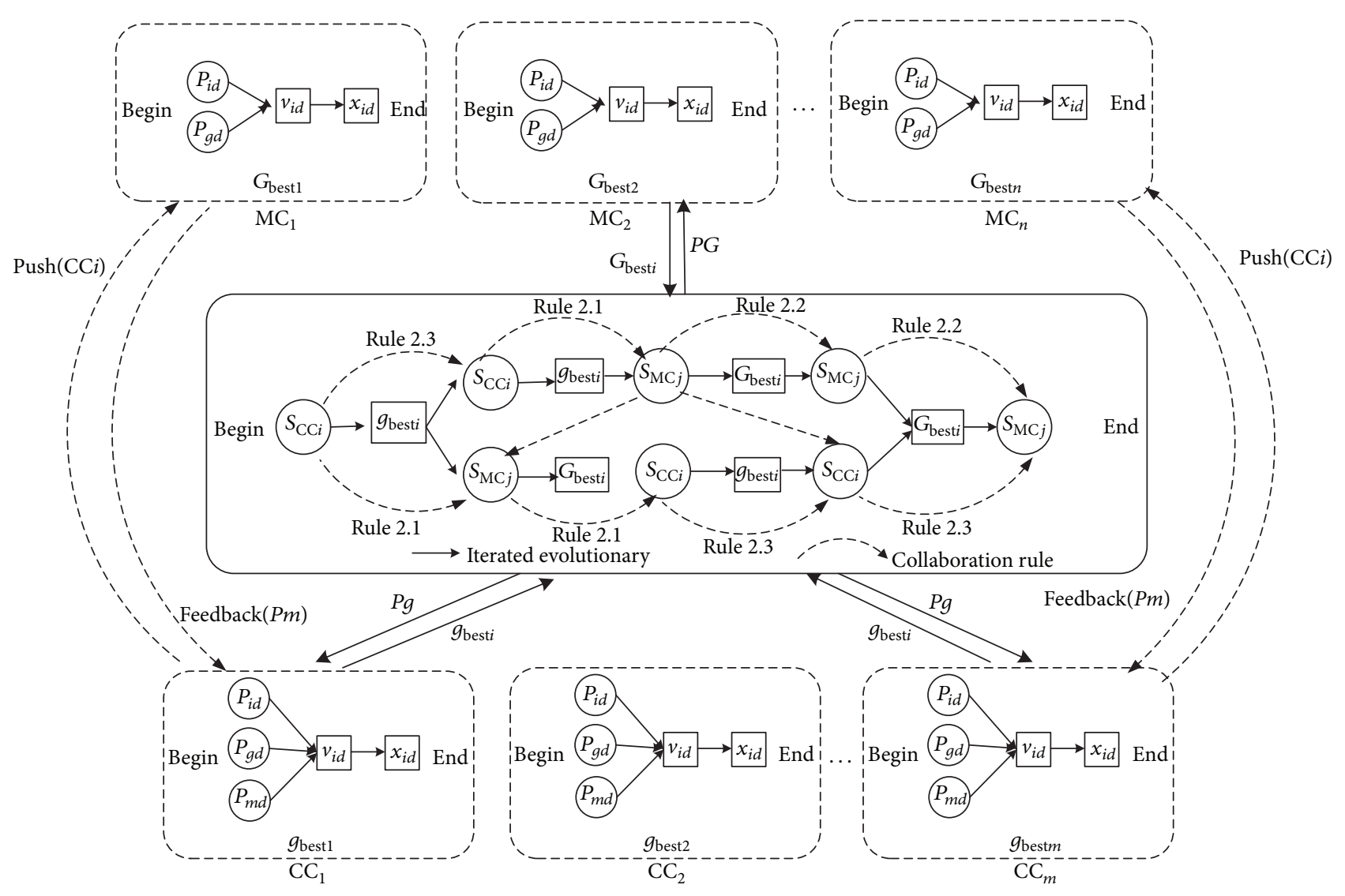

FIgURE 4: The multicommunity bidirectional drive mode.

into the evolution rules of the common communities to improve the searching ability. Thus, the asynchronous parallel implementation strategy for M-CBDCS is given, which could realize the parallel evolution within community and among communities. The designed searching strategy will benefit the overall optimization performance for RBF neural network.

Then, considering the influence of the network structure parameters on the validity of neural network approximating, the fitness function of parameters optimizing in M-CBDCS algorithm can be constructed as follows:

$$
f=\frac{1}{N} \sum_{n=1}^{N} \sum_{m=1}^{M}\left(y_{m n}-\widehat{y}_{m n}\right)^{2},
$$

where $y_{m n}$ is the practical output value of network after optimization; $\widehat{y}_{m n}$ is the desired output value of network; $N$ is the number of training samples; $M$ is the number of output neurons.

Particularly, the asynchronous parallel implementation strategy for RBF neural network is displayed in Figure 5. The $\mathrm{M}$-CBDCS is thought to regulate the essential parameters adaptively, where the structure parameters of RBF neural network are used as initial space. The nonlinear approximation error is obtained and serves as a novel leakage term in the adaptations to guarantee the sliding surface convergence stably and estimate the chattering phenomenon. The detailed implementation can be seen in the following steps.
Step 1 (initialization). Determine the RBF neural network topology including the number of hidden layer and neurons for each layer.

An initial population is selected from the structure parameters of RBF neural network. Assume the total number of particles in population is $n$; the number of communities is $q$; the iteration times, acceleration coefficients, and the inertia coefficient are initial set.

Step 2. According to the number of communities, the initial particles are evenly distributed to the processes and the community size is int $(n / q)$ at present. The remaining particles are randomly assigned to processes after rounding.

Step 3. Calculate the fitness of each particle in communities by (28).

Step 4. Calculate the fitness value of populations and divide the populations into two groups according to the decision threshold: model community and common community. Then construct the multicommunity cooperation network.

Step 5. Update the position and velocity of particles according to the multicommunity bidirectional drive rules.

Step 6. Update the global optimal value from model communities. 


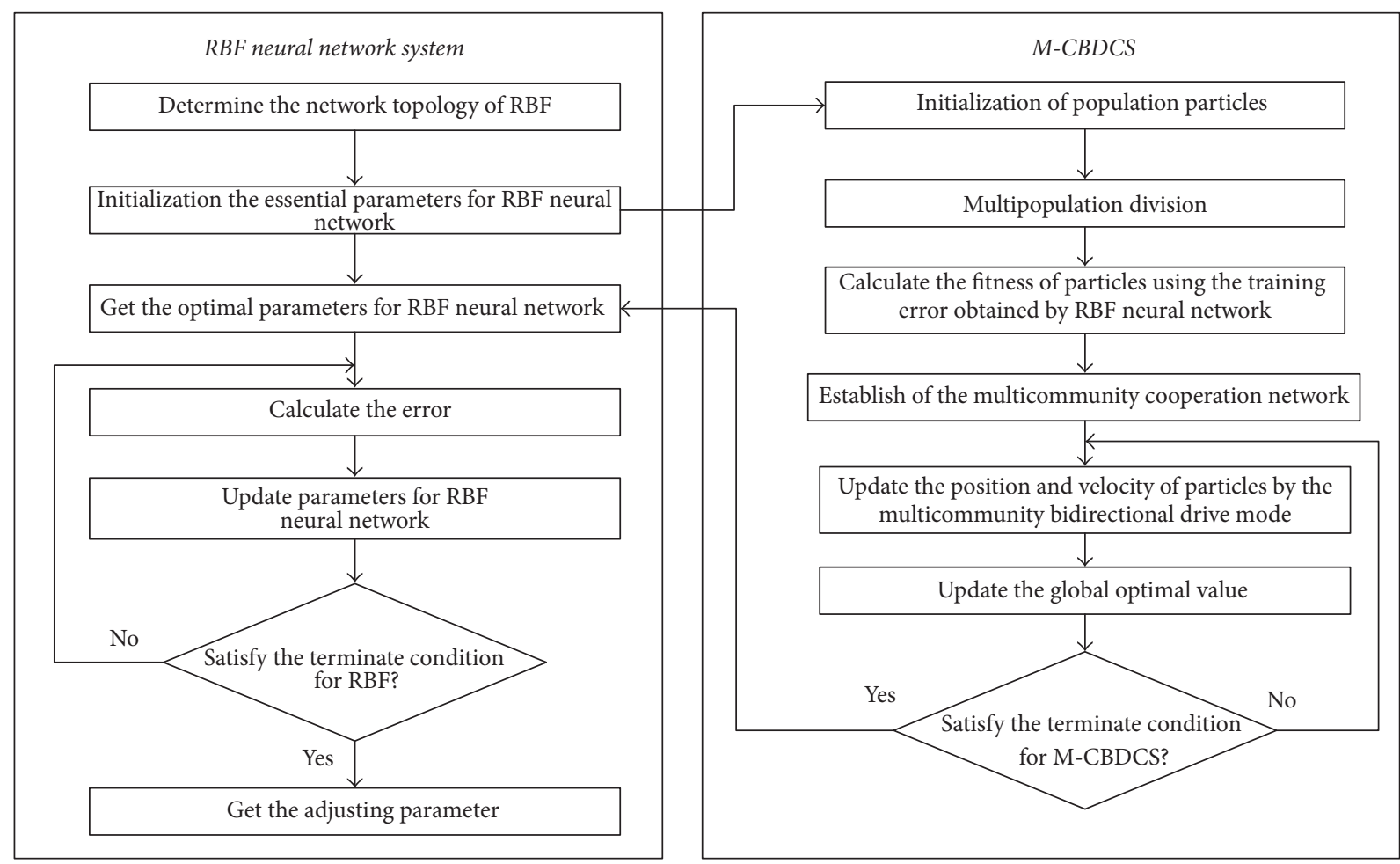

FIgURE 5: The flow diagram about the adaptive RBF neural network with the optimization of M-CBDCS.

Step 7. Loop to the Step 5 until the iterative termination condition of M-CBDCS is met.

Step 8. Get the optimal parameters for RBF neural network from M-CBDCS.

Step 9. Calculate the error between the actual output value and the desired output value of network.

Step 10. Update the parameters for RBF neural network according to the error above.

Step 11. Loop to Step 9 until the iterative termination condition of RBF neural network is met. And we can get the adaptive adjusting parameter for sliding mode control.

Figure 5 displays the flow chart of the adaptive RBF neural network under the optimization of M-CBDCS.

\subsubsection{Adaptive Neural Network Sliding Mode Control for} QTRA Attitude. The block diagram of the designed adaptive neural network sliding mode control structure is shown in Figure 6. In this diagram, the advantage of M-CBDCS is to adjust the value of the three key parameters adaptively, which can enhance the ability of nonlinear approximation and accelerate the convergence speed for RBF neural network. Similarly, the adaptive RBF neural network is used to generate the adaptive adjustment factor in sliding mode control to reduce the chattering phenomenon.
TABLE 1: Parameters for experiment.

\begin{tabular}{lc}
\hline Parameter & Value \\
\hline Mass for QTRA $(\mathrm{kg})$ & 6.25 \\
$I_{x x}\left(\mathrm{~kg} \cdot \mathrm{m}^{2}\right)$ & 4.568 \\
$I_{y y}\left(\mathrm{~kg} \cdot \mathrm{m}^{2}\right)$ & 3.109 \\
$I_{z z}\left(\mathrm{~kg} \cdot \mathrm{m}^{2}\right)$ & 2.796 \\
$I_{x z}\left(\mathrm{~kg} \cdot \mathrm{m}^{2}\right)$ & 0.028 \\
\hline
\end{tabular}

\section{Simulations}

In this section, in order to verify the effectiveness of the controller design algorithm proposed in this paper, simulations by using Matlab/Simulink for three control schemes are performed: (1) the adaptive neural network sliding mode controller (ANNSMC) proposed in this paper; (2) the classical neural network sliding mode controller (CNNSMC) proposed in [30]; (3) the dynamic inverse PID controller (DIPID) proposed in [31].

The designed controller is applied to the attitude control of the QTRA with respect to different reference signals: step signal, random noise, external disturbances, and hybrid superposition signal. And the parameters for the experiment are shown in Table 1.

Case 1 (step response). Considering that the landing process of QTRA usually adopts the method of equal angle descent, 


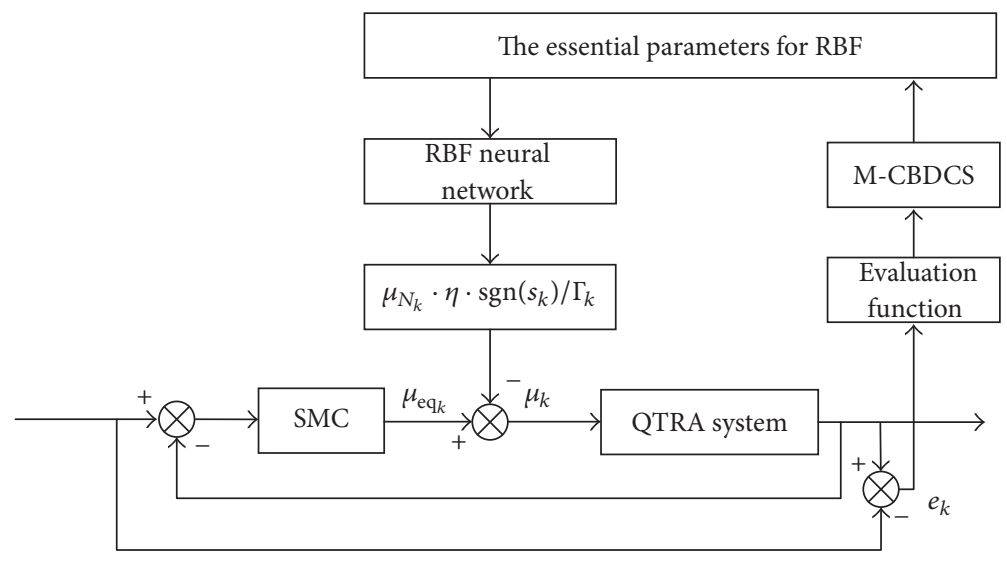

FIGURE 6: The control structure of the adaptive neural network sliding mode controller for QTRA.

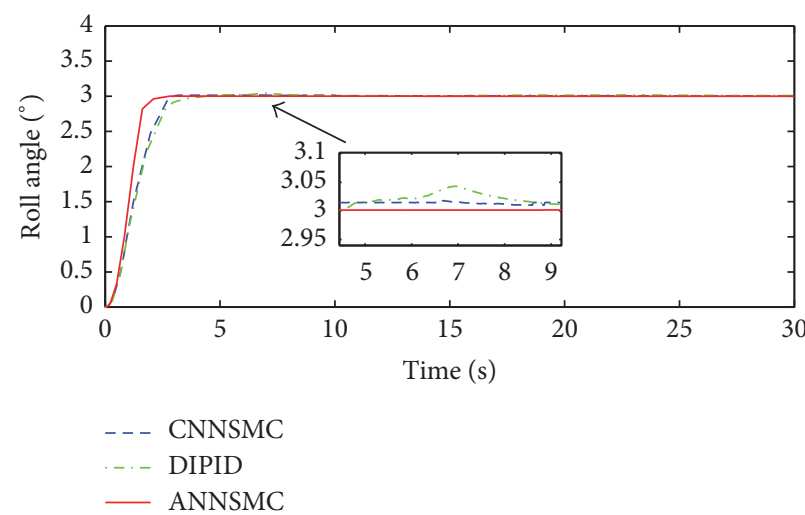

(a)

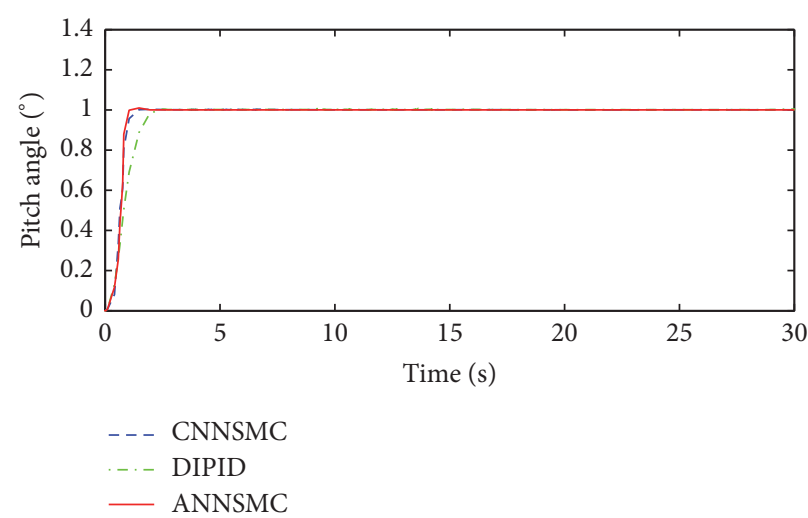

(b)

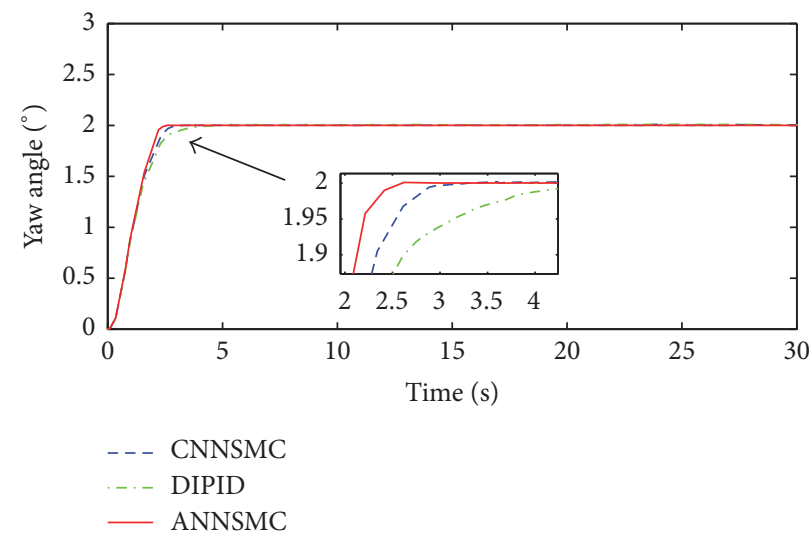

(c)

FiguRE 7: Helicopter attitude behavior response to the step signal.

the step signal is used to verify the control performance of these controllers for steady tracking in the simulation. We start the aircraft at the attitude $(\phi, \theta, \varphi)=(0,0,0)$ and the aircraft has the task of performing hover flight at $(\phi, \theta, \varphi)=$ $\left(3^{\circ}, 1^{\circ}, 2^{\circ}\right)$. The evolution and convergence of states $(\phi, \theta, \varphi)$ to the desired reference with the initial conditions are shown in Figure 7.

The results show that the method presented in this paper can eliminate steady state errors effectively with the fast convergence speed. But the other methods about CNNSMC and DIPID have a certain fluctuation in the process of tracking instruction with poor steady state performance.

Case 2 (random noise). Normally this simulation is designed to observe the effects of a white Gaussian random noise, which is generated with a maximum amplitude of 0.01 . In this case, the disturbance in the feedback loops is to simulate the sensor noise. And the simulation results show the comparison of adaptive controller versus other controllers 


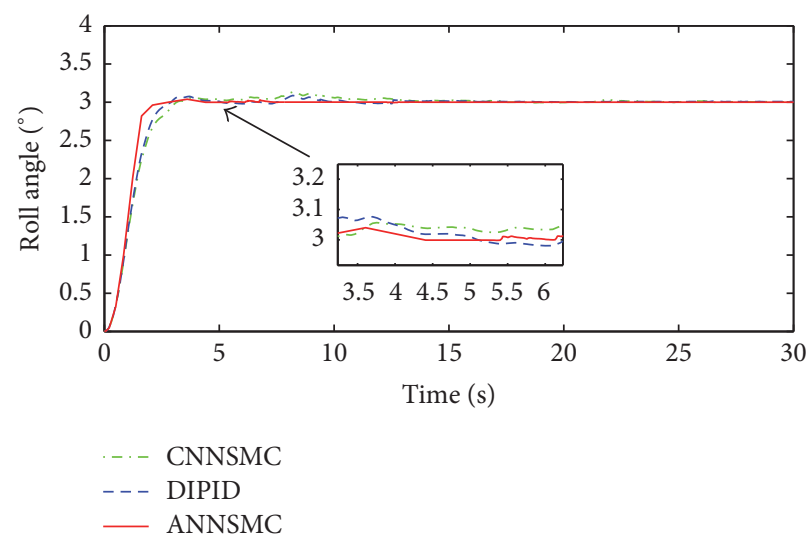

(a)

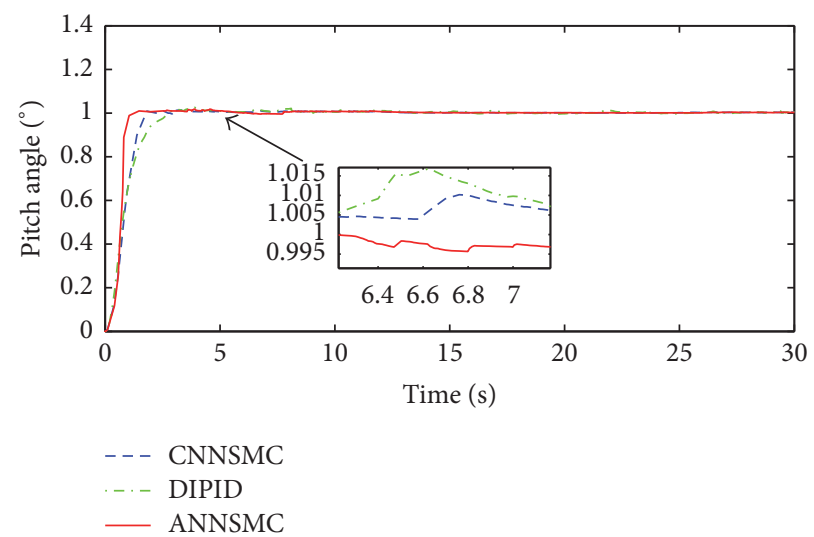

(b)

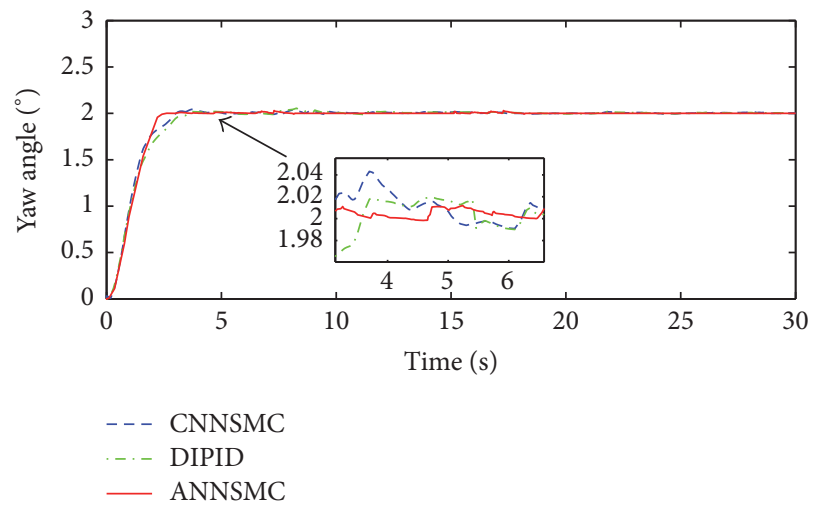

(c)

FIgURE 8: Helicopter attitude behavior response to the step signal with random noise.

in Figure 8, which implied that the ANNSMC controller has a better capability of suppressing noise than the other two methods. It can be concluded that the adaptive learned upper bound of noise can reduce chattering significantly with the introduction of M-CBDCS. And then a small boundary layer about switching surface can be generated by using the adaptive sliding mode control. Consequently, the ANNSMC controller gives a smooth control and proves to be effective for attitude control of QTRA.

Case 3 (external disturbances). In the actual system, the parameter perturbation and external disturbance are inevitable. In order to further verify the anti-interference performance of the ANNSMC controller, the external disturbances test is carried in this section. As we can see form Figure 9, the capacity of the presented controller guarantees a more steady flight of the airframe.

Case 4 (dynamic tracking). To verify the tracking capacity for the proposed method, one more simulation is run such that the desired reference is given using a hybrid superposition signal. The simulation results indicate that the maximum tracking error percentage in the attitude of QTRA is approximately $1.89 \%$ as shown in Figure 10 . It is important to note that angle references are tracked with negligible steady state errors.
In summary, the control strategy proposed in this paper has good performance for QTRA attitude control. Results in Case 1 show that the presented control strategy has relatively lesser settling time in the attitude control performance compared to other controllers. And Cases 2, 3, and 4 further verify the robustness and stability of the designed controller.

\section{Conclusion}

This paper describes an adaptive online control of attitude angles for nonlinear QTRA systems. The attitude dynamics of this aircraft including uncertain external disturbance are derived at VTOL mode. The proposed control strategies were evaluated by appropriate comparisons showing satisfactory results. A novel RBF neural network sliding mode control system was designed and built to implement an attitude control law. The presented adaptive RBF neural network is based on a multicommunity bidirectional drive collaborative search algorithm since it is suitable for implementation purposes with powerful parallel computing capacity. The comparative simulations between the proposed control method and classical neural network sliding mode control and dynamic inverse PID control were designed to simulate an attitude stabilized flight in VTOL stage with external disturbances.

Future work includes the transition flight between cruise and hover flight modes to evaluate the accuracy of the control 


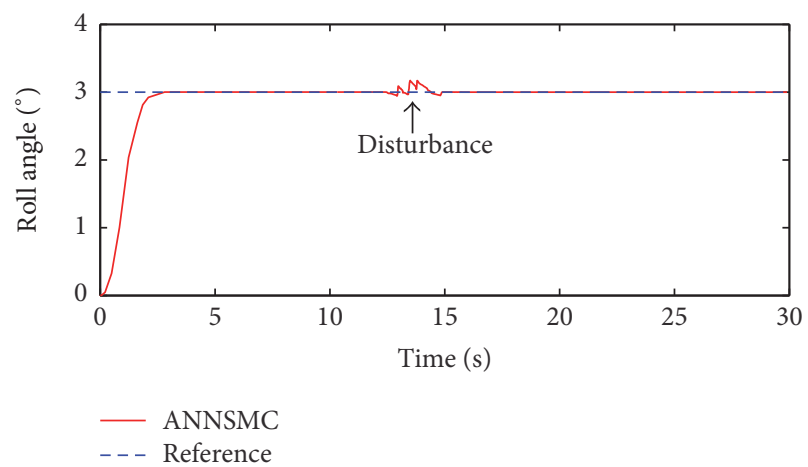

(a)

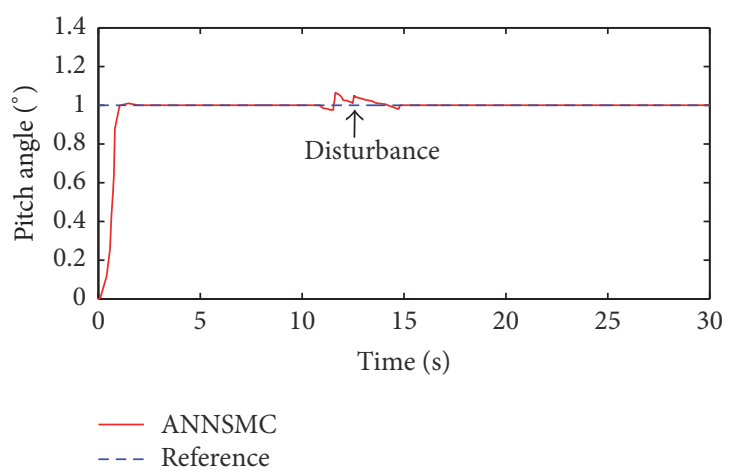

(b)

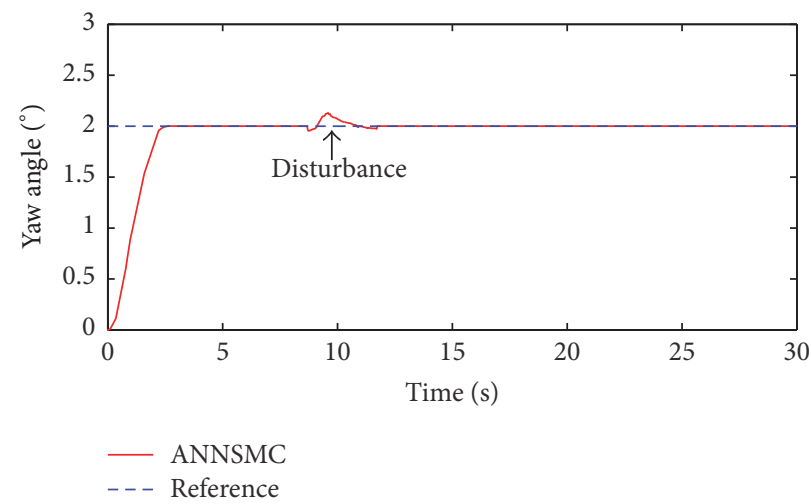

(c)

FIGURE 9: Helicopter attitude behavior response to the step signal with external disturbances.
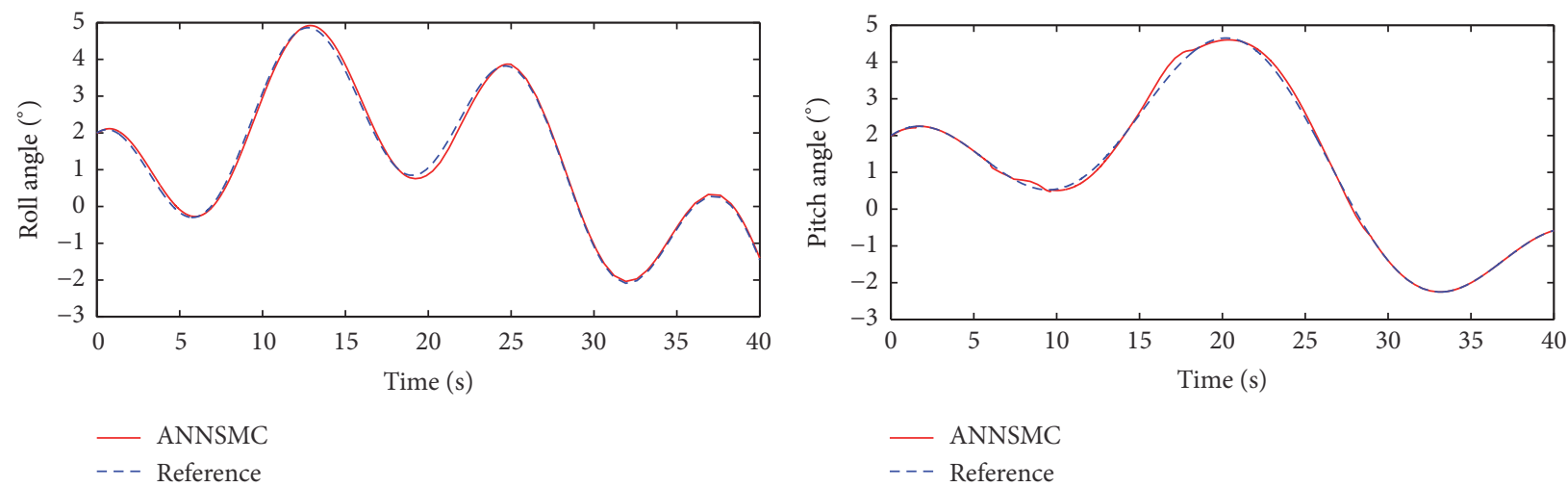

(a)

(b)

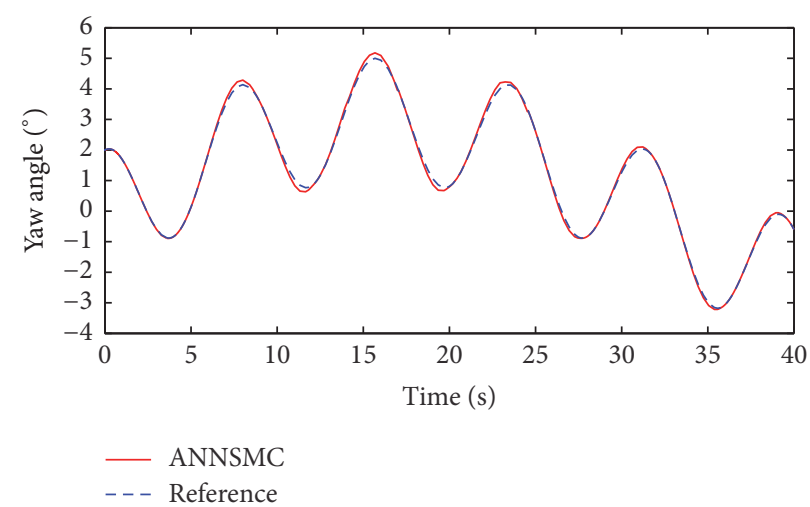

(c)

FIGURE 10: Helicopter attitude behavior response to the hybrid superposition signal. 
model to validate the control algorithm and the development of experimental convertible aircraft to implement the proposed design and configuration.

\section{Conflicts of Interest}

The authors declare that there are no conflicts of interest regarding the publication of this paper.

\section{Acknowledgments}

This paper was supported by the National Natural Science Foundation of China (51365022) and the Science Research Foundation Project of Education Department of Yunnan Province (2016YJS022).

\section{References}

[1] S. Abdulkerim Fatih and e. Erdinc, "Erdinc, altugsystem, design of a novel tilt-roll rotor quadrotor UAV," Journal of Intelligent Robotic Systems, vol. 84, Article ID 575599, pp. 575-599, 2016.

[2] X. Wang, "Takeoff/landing control based on acceleration measurements for VTOL aircraft," Journal of the Franklin Institute, vol. 350, no. 10, pp. 3045-3063, 2013.

[3] T. Oktay and C. Sultan, "Modeling and control of a helicopter slung-load system," Aerospace Science and Technology, vol. 29, no. 1, pp. 206-222, 2013.

[4] C. S. Lee and R. V. Gonzalez, "Fuzzy logic versus a PID controller for position control of a muscle-like actuated arm," Journal of Mechanical Science and Technology, vol. 22, no. 8, pp. 1475-1482, 2008.

[5] J. Na, G. Herrmann, and K. Zhang, "Improving transient performance of adaptive control via a modified reference model and novel adaption," International Journal of Robust and Nonlinear Control, vol. 27, no. 8, pp. 1351-1372, 2017.

[6] J. Li and Y. Li, "Dynamic analysis and PID control for a quadrotor," in Proceedings of International Conference on Mechatronics and Automation, (ICMA '11), pp. 573-578, August 2011.

[7] A. Das, F. Lewis, and K. Subbarao, "Backstepping approach for controlling a quadrotor using lagrange form dynamics," Journal of Intelligent and Robotic Systems, vol. 56, no. 1, pp. 127-151, 2009.

[8] A. Scottedward Hodel and C. E. Hall, "Variable-structure PID control to prevent integrator windup," IEEE Transactions on Industrial Electronics, vol. 48, no. 2, pp. 442-451, 2001.

[9] H.-B. Shin and J.-G. Park, "Anti-windup PID controller with integral state predictor for variable-speed motor drives," IEEE Transactions on Industrial Electronics, vol. 59, no. 3, pp. 15091516, 2012.

[10] C. Peng, Y. Bai, G. Qiao, X. Gong, and Y. Tian, "Anti-windup and multi-mode PID control of yaw movement for a quad-rotor UAV," Robot, vol. 37, no. 4, pp. 415-423, 2015.

[11] J.-B. Song, Y.-S. Byun, J. Kim, and B.-S. Kang, "Guidance and control of a scaled-down quad tilt prop PAV," Journal of Mechanical Science and Technology, vol. 29, no. 2, pp. 807-825, 2015.

[12] J. Na, M. N. Mahyuddin, G. Herrmann, X. Ren, and P. Barber, "Robust adaptive finite-time parameter estimation and control for robotic systems," International Journal of Robust and Nonlinear Control, vol. 25, no. 16, pp. 3045-3071, 2015.
[13] J.-J. Xiong and E.-H. Zheng, "Position and attitude tracking control for a quadrotor UAV," ISA Transactions, vol. 53, no. 3, pp. 725-731, 2014.

[14] B.-T. Zhang, F.-R. Gao, and K. Yao, "Neural network and adaptive algorithm-based fractional order sliding mode controller," Control Theory and Applications, vol. 33, no. 10, pp. 1373-1377, 2016.

[15] R.-J. Wai, "Fuzzy sliding-mode control using adaptive tuning technique," IEEE Transactions on Industrial Electronics, vol. 54, no. 1, pp. 78-87, 2007.

[16] C. Yang, Z. Li, R. Cui, and B. Xu, "Neural network-based motion control of an underactuated wheeled inverted pendulum model," IEEE Transactions on Neural Networks and Learning Systems, vol. 25, no. 11, pp. 2004-2016, 2014.

[17] J. Na, Q. Chen, X. Ren, and Y. Guo, "Adaptive prescribed performance motion control of servo mechanisms with friction compensation," IEEE Transactions on Industrial Electronics, vol. 61, no. 1, pp. 486-494, 2014.

[18] Y.-A. Zhang, M.-Z. Yu, and H.-L. Wu, "Sliding mode synchronization of fractional-order chaotic systems based on adaptive neural network," Control and Decision, vol. 30, no. 5, pp. 882886, 2015.

[19] P. Li, J. J. Ma, W. Q. Li, and Z. Q. Zheng, "Improved integral sliding mode control for a class of nonlinear uncertain systems," Control and Decision, vol. 24, no. 10, pp. 1463-1472, 2009.

[20] C. Yang, X. Wang, Z. Li, Y. Li, and C. Su, "Teleoperation control based on combination of wave variable and neural networks," IEEE Transactions on Systems, Man, and Cybernetics: Systems, 2017.

[21] S. Seshagiri and H. K. Khalil, "Robust output feedback regulation of minimum-phase nonlinear systems using conditional integrators," Automatica, vol. 41, no. 1, pp. 43-54, 2005.

[22] J. H. Wang and J. B. Hu, "Adaptive backstepping higher-order terminal sliding mode control for uncertain nonlinear systems," Control and Decision, vol. 27, no. 3, pp. 413-418, 2012.

[23] K. Mathiyalagan and K. Balachandran, "Finite-time stability of fractional-order stochastic singular systems with time delay and white noise," Complexity, vol. 21, no. S2, pp. 370-379, 2016.

[24] S. Li, H. Du, and X. Yu, "Discrete-time terminal sliding mode control systems based on Euler's discretization," Institute of Electrical and Electronics Engineers. Transactions on Automatic Control, vol. 5, no. 2, pp. 546-552, 2014.

[25] J. Zhu, S. Yakun, L. Hongyi, and L. Li, "Tracking control of uncertain switched nonlinear cascade systems: a nonlinear $\mathrm{H}$ $\infty$ sliding mode control method," Nonlinear Dynamics, vol. 73, no. 8, pp. 1803-1812, 2014.

[26] Y. Yin, H. Niu, and B. Chang, "Hongwei Niu, BinLei Chang, Multi-community bidirectional drive collaborative search algorithm," Computer Integrated Manufacturing Systems, vol. 23, no. 7, pp. 1581-1592, 2017.

[27] Y. Yin and Y. Huang, "Method of product innovation acquisition based on dynamic response particle swarm optimization," Advanced Materials Research, vol. 542-543, pp. 3-7, 2012.

[28] C. Yang, Y. Jiang, Z. Li, W. He, and C.-Y. Su, "Neural control of bimanual robots with guaranteed global stability and motion precision," IEEE Transactions on Industrial Informatics, vol. 13, no. 3, pp. 1162-1171, 2017.

[29] A. F. Şenkul and E. Altuğ, "System design of a novel Tilt-roll rotor quadrotor UAV," Journal of Intelligent and Robotic Systems: Theory and Applications, vol. 84, pp. 1-25, 2016. 
[30] H. J. Jayakrishnan, "Position and attitude control of a quadrotor UAV using super twisting sliding mode," IFAC-PapersOnLine, vol. 49, no. 1, pp. 284-289, 2016.

[31] A. Alkamachi and E. Erçelebi, "Modelling and genetic algorithm based-PID control of $\mathrm{H}$-shaped racing quadcopter," Arabian Journal for Science \& Engineering, pp. 1-10, 2017. 


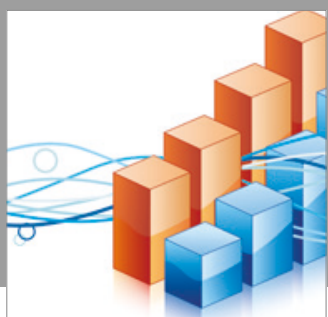

Advances in

Operations Research

vatersals

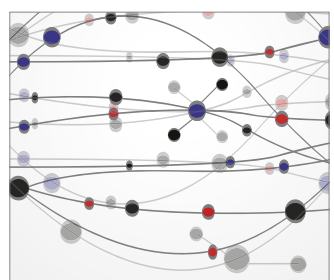

\section{The Scientific} World Journal
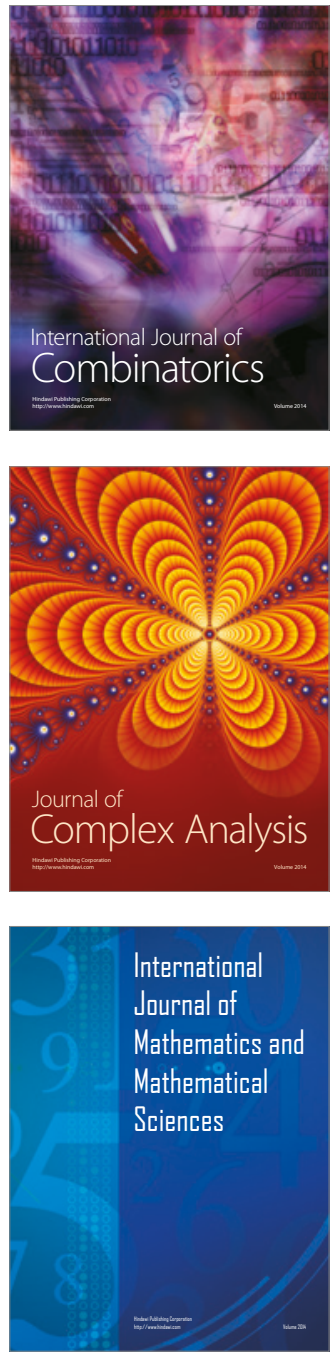
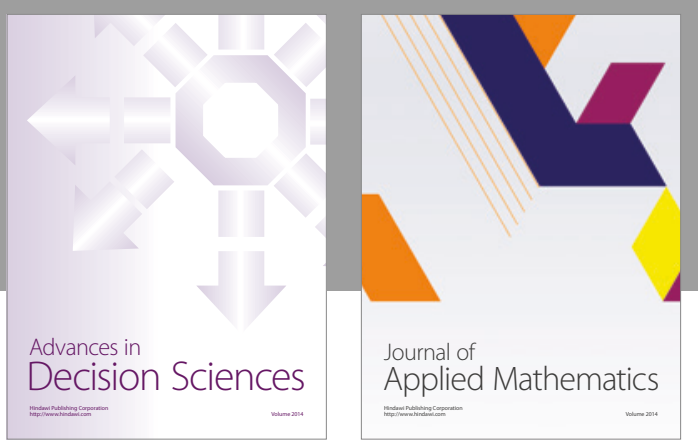

Algebra

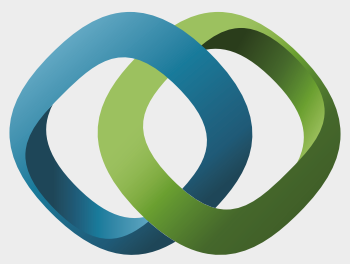

\section{Hindawi}

Submit your manuscripts at

https://www.hindawi.com
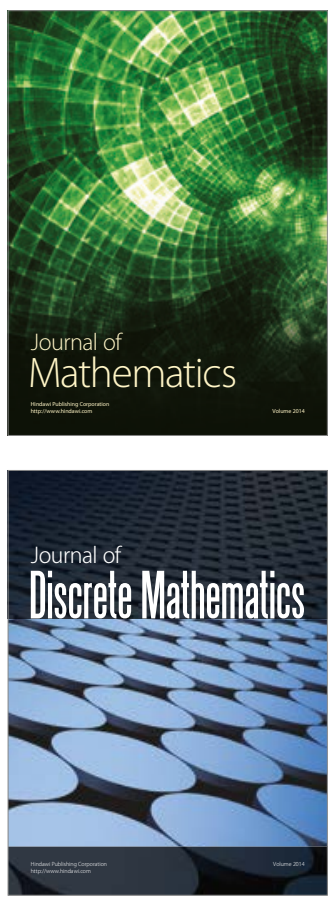

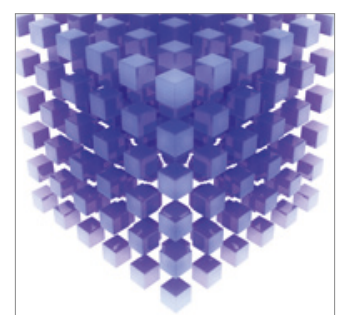

Mathematical Problems in Engineering
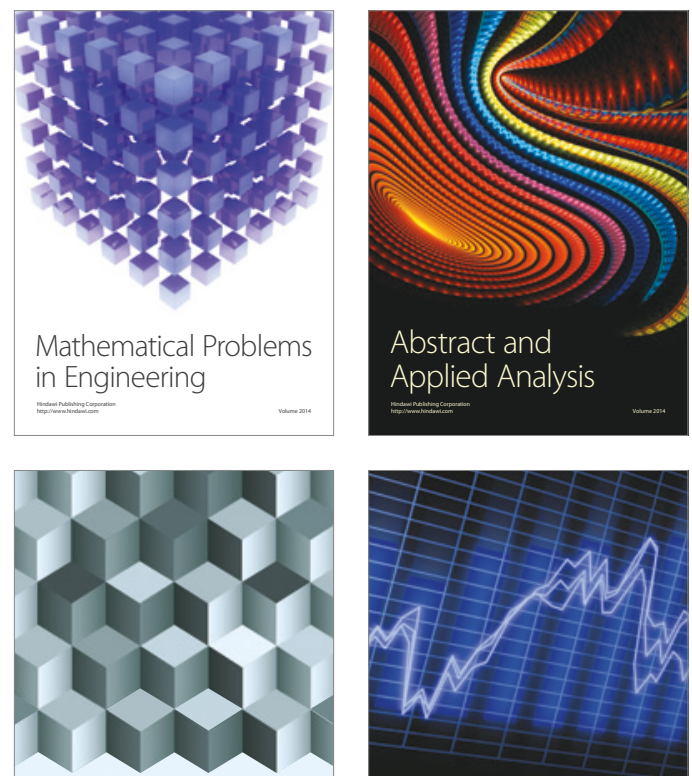

Journal of

Function Spaces

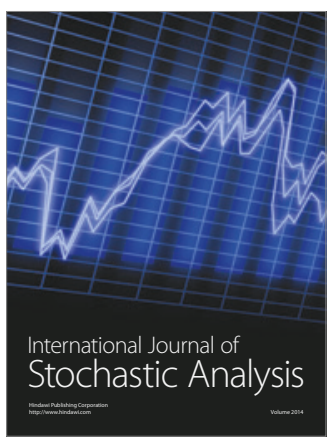

Probability and Statistics
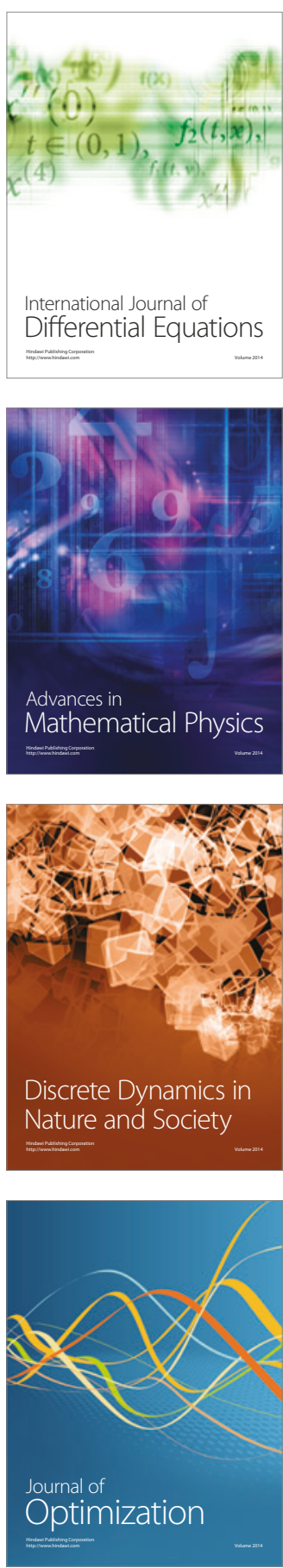